\title{
ANOMIA Y FATALISMO \\ EN LA TEORÍA DURKHEIMIANA \\ DE LA REGULACIÓN
}

\section{Philippe Besnard*}

\author{
CNRS, París
}

\begin{abstract}
RESUMEN
En El Suicidio, la teoría de la regulación es incompleta a causa de la marginación que sufre el tipo de suicidio fatalista. Se propone una nueva lectura de la obra orientada a reconstruir el concepto de fatalismo y depurar el concepto de anomia. El resultado es la emergencia de una teoría sistemática y conceptualmente rigurosa que permite distinguir cuatro tipos de regulación patológica: fatalismo agudo, fatalismo crónico, anomia aguda y anomia crónica. Esta propuesta de reconceptualización se contrapone a otras que no resuelven los problemas de lectura que El suicidio comporta.
\end{abstract}

La palabra 'anomia' ha seguido una trayectoria extraña. Acuñada por JeanMarie Guyau (1885) como un juego de palabras sobre el término kantiano 'autonomía', Durkheim la incorporó al vocabulario de la sociología naciente. La noción de Durkheim fue redescubierta en los años treinta en la Universidad de Harvard. En los sesenta se convirtió en el nombre de una "medida", ejemplo de los procedimientos de investigación preponderantes en la sociología americana (Besnard, 1987). Su carrera terminó en la mayor confusión. La utilización principal de la palabra 'anomia' ha sufrido una completa revolución

* S. Turner (ed.), Emile Durkheim. Sociologist and Moralist, London, Routledge, 1993, pp. 169-190. 
semántica: su significado final es la antítesis del de Durkheim. Todavía la inmensa mayoría de los que la emplean suponen simplemente que el concepto no ha cambiado desde la época de Durkheim.

Desde sus orígenes, la palabra anomia ha estado rodeada de una confusión conceptual. La metamorfosis semántica sufrida, que argumentaremos aquí, está arraigada en la oscuridad relativa del concepto en los propios escritos de Durkheim. La noción de anomia está, sorprendentemente, mal elaborada en su trabajo. Por lo tanto, si deseamos establecer el contenido específicamente durkheimiano del concepto, debemos depurarlo de las connotaciones secundarias y parásitas que lo estorban y liberarlo de la red de conceptos en que está enmarañado. Pero esto no basta para aclararlo, ya que la propia presentación de Durkheim contribuye a la confusión. Por ejemplo, en La división del trabajo social (1893), "la división anómica del trabajo» ni se sitúa ni se define en relación con las otras formas patológicas de la división del trabajo. La anomia se concibe como la ausencia o la defectuosidad de la regulación social necesaria para provocar la cooperación entre funciones especializadas. Pero al final del capítulo que dedica a esta forma anómica, Durkheim trata de una patología de las sociedades industriales diferente: la alienación del trabajador que efectúa tareas fragmentadas. Numerosos lectores de Durkheim concluyeron, lo que es comprensible, que la falta de sentido del trabajo fragmentado es la anomia. Tal interpretación es errónea. La alienación del trabajador muy especializado, como Durkheim la concibe, es una situación no sólo diferente, sino realmente contraria de la anomia.

El tema de la anomia como una enfermedad permanente de las sociedades industriales está más desarrollado en El Suicidio (1897). Si queremos definir, de la manera más estricta posible, lo que implica la noción de suicidio anómico, necesitamos hacer cuidadosas distinciones. Para empezar, hay que aceptar la distinción etiológica que Durkheim establece entre suicidio egoísta y suicidio anómico, y la independencia de las dos variables, integración y regulación, que utiliza para explicar las variaciones en el suicidio. La mayoría de los que han escrito sobre El Suicidio dudan que esta distinción sea apropiada. Sin embargo, tiene un fundamento conceptual y otro empírico (Besnard, 1987: 6281). El énfasis en ambos aspectos de la relación entre el individuo y la sociedad no es exclusivo de El Suicidio. También se encuentra en la Educación moral (1925), donde Durkheim diferencia «el espíritu de disciplina» y "la adhesión a los grupos sociales». La confusión entre anomia y egoísmo es consecuencia de este emparejamiento frecuente. Lo que también explica por qué Durkheim ha sido presentado tan a menudo como "el sociólogo de la anomia». Quiero demostrar aquí que es un error: la anomia no es más que un tema menor, tratado de pasada en la obra de Durkheim; más aún, un tema apenas elaborado.

Una de las peculiaridades de El Suicidio es el contraste entre el tratamiento de las dos variables independientes, integración y regulación, por parte de Durkheim. La teoría de la integración es mucho más coherente y completa que 
la teoría de la regulación. Se dice que un grupo social está integrado en la medida en que sus miembros: 1) posean una conciencia común y compartan los mismos sentimientos, creencias y prácticas; 2) interaccionen entre sí; y 3) sientan perseguir metas comunes. Cada uno de sus tres ejemplos, a saber, la sociedad religiosa, la sociedad doméstica y la sociedad política, aporta una ilustración de uno de los tres componentes. La teoría de la integración vincula los sentimientos colectivos al grado de interacción social y, de esta forma, a la estructura morfológica de la sociedad, como en La división del trabajo social, pero, por supuesto, la discusión se dedica totalmente a las dos situaciones, egoísmo y altruismo, definidas por los valores extremos de las variables.

La teoría de la regulación se desarrolla mucho menos. La reconstrucción de la teoría de la regulación de Durkheim es el aspecto más difícil de la interpretación de El Suicidio. Debemos situar la anomia en el contexto de la regulación y elucidar su relación con su pretendido homólogo, el fatalismo. Pero Durkheim hizo todos los esfuerzos por minimizar la importancia del suicidio fatalista, aportando ilustraciones empíricas no muy convincentes. Sin embargo, en lo que concierne a la regulación, poseía realmente los datos empíricos requeridos - lo que no era el caso de la integración - necesarios para la construcción de una teoría coherente y completa, cuya ausencia es, en efecto, la mayor peculiaridad de El Suicidio.

\section{LA OCULTACIÓN DEL FATALISMO}

El esfuerzo de Durkheim por relegar el suicidio fatalista a un segundo plano tuvo éxito: numerosos comentaristas han olvidado este cuarto tipo de suicidio. Durkheim lo trata directamente en una nota a pie de página al final del capítulo sobre el suicidio anómico, y da algunos ejemplos que ya ha considerado. Dice así:

«Las consideraciones precedentes muestran que existe un tercer tipo de suicidio opuesto al suicidio anómico, como el suicidio egoísta y el suicidio altruista se oponen entre sí. Resultado de un exceso de reglamentación, es el que comenten los sujetos cuyo futuro está despiadadamente cerrado, cuyas pasiones están violentamente reprimidas por una disciplina opresiva. Es el suicidio de los maridos demasiado jóvenes, de la mujer casada sin hijos. Para ser exhaustivos deberíamos crear un cuarto tipo de suicidio. Pero tiene tan poca importancia hoy y, exceptuando los casos que acabamos de citar, es tan difícil encontrar ejemplos que nos parece inútil fijarnos en él. Sin embargo, podría tener un interés histórico. ¿No están vinculados a este tipo los suicidios de esclavos aparentemente tan frecuentes en ciertas condiciones (...) y todos los que, en una palabra, pueden atribuirse a las intemperancias del despotismo material o moral? Para destacar este carácter ineluctable e inflexible de la regla contra la 
que nada se puede hacer, y por oposición a este término de anomia que acabamos de emplear, podríamos llamarlo el suicidio fatalista» (1897: $311)$.

A primera vista, parece extraño que Durkheim dé tan poca importancia a este cuarto tipo de suicidio. Su falta de interés se comprende mejor si recordamos que el texto al que se adjunta esta nota trata de la anomia conyugal. A través del estudio de esta forma de regulación, Durkheim revela sin duda la posibilidad de que la regulación social excesiva pueda ser patógena. Pero su principal interés era las implicaciones sobre el suicidio del debilitamiento de la disciplina conyugal, que él mismo había observado. La ley de divorcio en Francia data de 1884 (tres años antes del propio matrimonio de Durkheim). Aunque esa ley simplemente sancionase un "debilitamiento de la regulación», el establecimiento legal del divorcio le parecía haber tenido claros efectos sobre la inestabilidad matrimonial (1897: 307).

Se trataba de un tema que le interesaba particularmente y sobre el que volvía con frecuencia, utilizando los resultados empíricos de El Suicidio. Un ejemplo de ello es un artículo (virtualmente un post scriptum a El Suicidio en algunos aspectos) publicado en la Revue Bleue (1906), en el que Durkheim adopta una postura pública en el debate sobre el divorcio por consentimiento mutuo iniciado por Paul y Victor Margueritte. En este artículo (analizado con más detalle en Besnard, 1973: 57-60), Durkheim afirma demostrar que la fragilidad del vínculo matrimonial es tan nociva para las mujeres casadas como para los hombres casados, aunque eso requiera malinterpretar las cifras. En consecuencia, mantiene que si la posibilidad del divorcio apenas tiene efecto en el suicidio femenino, se debe a una «ley más general»: «el estado del matrimonio sólo tiene un efecto débil en la constitución moral de las mujeres» (1906: 551). Esta afirmación audaz contradice la evidencia estadística de El Suicidio. Pero permite a Durkheim defender el carácter inofensivo del divorcio para las mujeres "porque el matrimonio es inoperante» (1906: 552). Esta propuesta le permitió fundamentar su oposición al divorcio por consentimiento mutuo sobre la base de que destroza la «regulación matrimonial» necesaria a «esta paz de espíritu, este equilibrio interno, que son condiciones esenciales de la salud moral y de la felicidad» (1906: 552). Durkheim utiliza la «felicidad de los cónyuges», incluyendo la felicidad de las viudas, como la base de su argumento contra la legalización de este "tipo de divorcio sui generis, que está separado de los otros tipos por un abismo" (1906: 552). Niega explícitamente el carácter distintivo del suicidio femenino e implícitamente la existencia misma del suicidio fatalista. Por lo tanto, no se puede exagerar la importancia del contexto histórico en el que Durkheim se encontró con el problema de la regulación social excesiva. El hecho de que lo tratara en su conexión con el divorcio le lleva probablemente a minimizar la importancia del fatalismo y, en consecuencia, a anularlo.

Las numerosas reseñas que publicó en L'Année Sociologique de libros o capí- 
tulos sobre el divorcio indican su interés especial por la cuestión y su deseo de sacar lecciones prácticas de esos estudios. En efecto, de todos los "problemas sociales» de la época de Durkheim, el divorcio es quizás al que volvió más a menudo y sobre el que adoptó la posición pública más clara. Sabía que esta posición implicaba el riesgo de, por error, «ser considerado un espíritu retrógrado", lo que no creía ser (1906: 549). Por lo tanto, no exageramos al decir que este problema particular jugó un papel decisivo en su tratamiento sociológico de la constricción social. Obsesionado por la relajación de la disciplina conyugal, apenas cuestionó «el debilitamiento de la regulación» en otras áreas de la vida social y parecía insensible al carácter "constrictivo» de las tendencias racionalizadoras de las sociedades modernas. A su entender, esas sociedades simplemente se dirigen hacia una regulación poco estricta, por lo que el suicidio fatalista sólo puede tener un «interés histórico».

Las convicciones de Durkheim sobre el futuro no justifican, sin embargo, la asombrosa desproporción entre esa nota y el capítulo entero dedicado al suicidio altruista. Después de todo, el único ejemplo «moderno» proporcionado en este capítulo es el suicidio militar, que en cierta manera podría igualmente interpretarse - hasta un cierto punto- en términos de regulación excesiva: Durkheim mismo se refiere en este capítulo a la "rígida» disciplina, que «reprime al individuo» (1897: 260). Aclara inmediatamente en una nota que no es la "represión» misma, sino más bien la falta de individualización, la que fomenta el suicidio. Pero nada se dice que sostenga esta afirmación. En un capítulo posterior del libro, cuando Durkheim considera otro ejemplo moderno del suicidio altruista, el suicidio de los presos, parece introducir dos factores en paralelo: la coherencia de la sociedad de los malhechores y presos, en la que «el individuo está completamente sumergido», y la "disciplina de la prisión» (1897: 391, n. 1).

El tratamiento del fatalismo está conectado con la modificación del marco de referencia de Durkheim, otra característica importante de El Suicidio. La concepción de la felicidad social e individual que se deriva de los primeros escritos de Durkheim puede describirse como una filosofía del término medio: nada que sea extremo o excesivo puede producir algo útil o agradable. Podemos decir con mayor precisión que esta concepción deriva del modelo de la curva en U (cuya influencia e importancia en La división del trabajo social se ha demostrado en Besnard, 1973: 33-34), evidente ya en la primera incursión de Durkheim en el tema del suicidio en 1888. De manera que, sorprendentemente, al tratar de la regulación social en El Suicidio, Durkheim sólo considera dos fuentes de patología social y hace todo lo posible por minimizar la importancia del suicidio fatalista que, a primera vista, es con respecto al suicidio anómico lo que la división forzada del trabajo respecto a la división anómica del trabajo. Esta falta de interés por el fatalismo revela una renuncia gradual a la curva en U como marco de referencia: la filosofía de la moderación entre los extremos es reemplazada por una filosofía del equilibrio entre fuerzas en conflicto.

No examinaremos con detalle en este texto esta transformación del marco 
de referencia intelectual que, además, está ligada a otros cambios en el enfoque de Durkheim e incluso en lo que se refiere a sus metáforas preferidas (cf. Besnard, 1973: 36-40). Baste decir que la totalidad de la obra pivota sobre el capítulo del suicidio anómico (capítulo 5 del libro II), punto en el que la teoría del término medio está siendo reemplazada por la teoría del equilibrio, y las referencias a las variables por las referencias a las tendencias. En el marco de esta situación ambigua, el modelo de la curva en U apenas se aplicó a la relación entre regulación social y suicidio. Al suicidio fatalista sólo se le dedicó una nota, lo que revela el abandono del antiguo marco de referencia. El ejemplo dado por Durkheim de las mujeres casadas sin hijos oculta la relación curvilineal que existe realmente entre regulación social y porcentaje de suicidios. En realidad, no sólo las mujeres casadas sin hijos, sino las mujeres casadas en general, experimentan las consecuencias de las obligaciones excesivas, aunque la presencia de hijos compense en parte el efecto nocivo de la disciplina matrimonial.

Esto facilita la comprensión del proceso lógico que le condujo a afirmar que el suicidio fatalista era "de poca importancia hoy" y que era "tan difícil encontrar ejemplos» que le parecía «inútil insistir en ello». El lugar que ocupa en esta obra la exposición de la regulación social, y la cuestión particular tratada, el divorcio, contribuyen a hacerle adoptar una posición que difiere en parte de su enfoque previo y más aún de sus propios datos empíricos. No había nada, en los datos originales usados por Durkheim, que justificara su clara preferencia por uno de los factores de la variación de los niveles de suicidio en detrimento del otro.

El hecho de que la existencia de esta variable bipolar (hiper- e hipo-regulación) esté implícita en los análisis empíricos de la anomia conyugal hace aún más injustificable la reducción de la variable de la regulación social a uno solo de sus polos, la hipo-regulación. Recordemos brevemente las líneas fundamentales de su análisis. Tras constatar que el número de suicidios y divorcios varía en paralelo de país a país, Durkheim, gracias a una brillante intuición, se fija en el efecto probable de la posibilidad de divorciarse sobre la "constitución matrimonial». Relaciona entonces el porcentaje de divorcios con el efecto del matrimonio, que no es el porcentaje de suicidios de los casados, sino su inmunidad en comparación con los solteros del mismo sexo. Así descubre que cuanto más frecuente es el divorcio, menor es la inmunidad de los maridos en relación con los solteros y mayor es la de las viudas en relación con las solteras. Dicho sea de paso, este resultado no implica en absoluto que las mujeres casadas se suiciden menos en los lugares donde el divorcio es frecuente que en aquellos en que es raro, aunque Durkheim mismo cayera en esta trampa bastantes veces.

Durkheim utiliza varios datos comparativos para apoyar este ejemplo; entre otros, los resultados de una comparación entre el Departamento del Sena y las provincias, a partir de datos originales sobre el suicidio en Francia de 1889 a 1891. En esta época, el divorcio era cuatro veces más común en el 
Departamento del Sena que en los otros. Así que estamos sobre seguro al apreciar el efecto del divorcio sobre la relación de las tasas de suicidio de los solteros, situada por encima de la tasa de los casados, especialmente si tomamos en cuenta la influencia de la edad [ver tabla 7, que indica las cifras de la tabla XXI de Durkheim (1897: 183)].

\section{TABLA 7}

Proporción del porcentaje de suicidio de solteros/porcentaje de suicidio de casados por sexo y edad, en provincias y en el Departamento del Sena

\begin{tabular}{|c|c|c|c|c|}
\hline \multirow[b]{2}{*}{ Edad } & \multicolumn{2}{|c|}{ Hombres } & \multicolumn{2}{|c|}{ Mujeres } \\
\hline & Provincias & Sena & Provincias & Sena \\
\hline $20-25$ & 2,25 & 3,80 & 1,82 & 3,06 \\
\hline 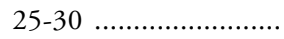 & 3,53 & 2,01 & 1,90 & 3,18 \\
\hline $30-40$ & 2,92 & 1,99 & 1,36 & 1,80 \\
\hline 40-50 ........................ & 3,30 & 1,21 & 1,54 & 1,64 \\
\hline 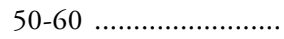 & 3,07 & 1,18 & 1,30 & 1,29 \\
\hline 60-70 …................... & 3,07 & 0,96 & 1,14 & 1,09 \\
\hline 70-80 ......................... & 3,01 & 1,02 & 1,04 & 0,48 \\
\hline 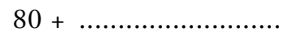 & 1,91 & 1,73 & 1,48 & 1,83 \\
\hline
\end{tabular}

La introducción de esta variable test de tipo ecológico confirma el resultado ya indicado por Durkheim: el matrimonio proporciona una mayor protección para el hombre allí donde el divorcio es escaso (en las provincias) que donde es frecuente (Sena). A la inversa, protege más a la mujer en París que en provincias. El efecto del contexto ecológico en la influencia del matrimonio sobre el suicidio no es, por lo tanto, el mismo para cada sexo. Este efecto interactivo puede formularse como sigue: el efecto del sexo en la influencia del matrimonio sobre el suicidio no es el mismo en París que en provincias. En realidad, en el Sena, a diferencia de Francia en su conjunto y de las provincias, la distancia entre casados y solteros no es mayor para los hombres que para las mujeres; en la mayoría de los grupos de edad es incluso menor. El medio urbano anula $-\mathrm{e}$ incluso tiende a invertir - la diferencia entre hombres y mujeres respecto a la inmunidad otorgada por el matrimonio, mientras que en provincias la diferencia se acentúa. Esto implica la existencia y acción de una variable de «relación conyugal» que tiene una relación curvilínea con el suicidio. Como el vínculo matrimonial es más débil en París que en provincias, el que en estas últimas la mujer casada esté menos protegida que en el Sena en comparación con las solteras (es decir, mayor fatalismo) y el que el marido esté más protegido en comparación con el soltero (anomia contenida de forma más eficaz) se ajusta a este modelo curvilineal. Esta relación del efecto del matrimonio con el sexo, la subcultura y el suicidio se expresa en el gráfico 7.1 


\section{GRÁFICO 7.1}

El efecto del matrimonio en relación con el sexo, la subcultura y el suicidio

ANOMIA

FATALISMO

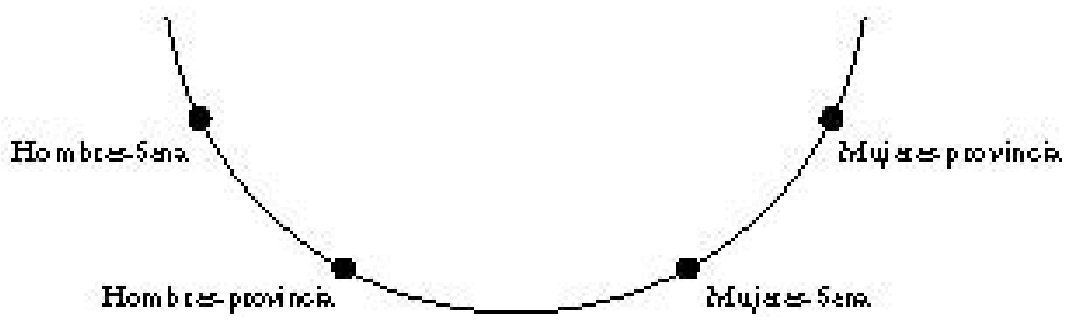

\section{LAS CONTRADICCIONES DEL FATALISMO: DESEO Y REGULACIÓN}

Al insistir en la anomia y minimizar el fatalismo, Durkheim no sólo no daba cuenta correctamente de los datos en su poder, sino que también ofrecía interpretaciones poco convincentes y, sobre todo, inadecuadas a su propio principio de buscar explicaciones sociales para las diferencias sociales. Es evidente respecto de la regulación matrimonial, así como en la explicación del efecto diferente de la obligación matrimonial sobre los dos sexos. Para dar cuenta de este "antagonismo entre los sexos», uno de los cuales "necesita obligaciones y el otro libertad» (1897: 309), Durkheim construye una teoría del deseo sexual. La naturaleza ilimitada de este deseo en el hombre va más allá del organismo, se «ha liberado parcialmente del cuerpo y casi parece haberse intelectualizado». El beneficio del matrimonio monógamo para el varón consiste en ser lo único capaz de proporcionar la regulación social indispensable para controlar las «inclinaciones» que ya no son una función de las «necesidades orgánicas». «El hecho de forzar al hombre a ligarse para siempre a la misma mujer, asigna un objeto a la necesidad de amar estrictamente definido y cierra el horizonte.» El hombre casado evita así el sufrimiento del «mórbido deseo de infinito que siempre acompaña a la anomia» (1897: 303-304). Al contrario, respecto a la mujer, «las necesidades sexuales tienen menos un carácter mental, porque, en general, su vida mental está menos desarrollada». Como es «una criatura más instintiva que el hombre, sólo tiene que seguir sus instintos para encontrar paz y tranquilidad» (1897: 306). El matrimonio monógamo, por lo tanto, no ayuda a la mujer a «limitar sus deseos», porque, a diferencia de los del hombre, «están naturalmente limitados». Como esta restricción carece de cometido, resulta excesiva. Durkheim se ve obligado en este caso a reconocer 
que la regulación social no produce, invariablemente, resultados apropiados. La disciplina, como en el matrimonio monógamo, "aun siendo útil, tiene sus inconvenientes (...) Al limitar el horizonte, cierra todas las salidas e impide todas las esperanzas, incluso aquellas legítimas» $(1897: 306)^{1}$.

Por lo tanto, Durkheim excluye finalmente a la mujer del campo de aplicación de la teoría de la anomia y explica la oposición entre los dos sexos por la dicotomía entre naturaleza y cultura o animalidad y humanidad. La anomia sexual no es más que una forma del ansia del infinito, que procede del hecho de que, a diferencia de los animales, el poder de regular las necesidades del hombre no forma parte de su composición orgánica. Las mujeres están, por tanto, excluidas del paraíso -o del infierno- del deseo y confinadas al limbo de lo subsocial y lo subhumano, junto con los niños y los viejos: «en ambos casos, el hombre físico tiende a convertirse en todo hombre» (1897: 230). Además, como para los niños, los ancianos y las mujeres la sociedad es una noción menos intensa, están menos predispuestos al suicidio. Durkheim no olvida aclarar que "la inmunidad del animal obedece a las mismas causas» (1897: 231).

Podríamos citar otros muchos ejemplos de los escritos de Durkheim para demostrar que, en su opinión, el hombre y la mujer no son, tal y como estaban las cosas entonces, "criaturas de la misma naturaleza» (1897: 444; cf. Besnard, 1973: 30-33). Por otra parte, Durkheim recurre, en efecto, a la diferencia «natural» entre los sexos para explicar el antagonismo de sus intereses en la institución del matrimonio. Es una explicación sorprendente viniendo de alguien que ha tratado de reducir todos los factores no sociales de variación del suicidio a causas sociales. Después de esforzarse, en lo que concierne a la influencia del sexo, en reducir la diferencia biológica a la diferencia social, termina por dar cuenta de las variaciones sociales recurriendo a la biología. En opinión de Durkheim, es indudable que la diferencia social no se reduce a la diferencia orgánica, pero el organismo es una de las precondiciones de la diferencia social.

En todo caso, se pueden detectar en El Suicidio las semillas de otra interpretación más sencilla y más sociológica de la situación de los dos sexos en relación con el matrimonio. Durkheim comenta de pasada que la moral otorga ciertos privilegios a los hombres, mientras que para las mujeres la «monogamia es una obligación estricta sin ninguna reserva» (1897: 306) ${ }^{2}$. Pero el que Durkheim no desarrolle este punto revela, una vez más, su olvido del fatalismo y su abandono del modelo curvilineal para el estudio de la relación entre la regu-

${ }^{1}$ Nos preguntarnos qué quiere decir Durkheim cuando habla de esperanzas legítimas con respecto a la libertad conyugal.

2 Posteriormente, durante el debate sobre el matrimonio y el divorcio citado más arriba, Durkheim (1909: 279) acentúa sobre todo esta segunda interpretación: para las mujeres, «el instinto sexual ya está contenido y moderado, incluso fuera del matrimonio, por la moral y la opinión, que, a este respecto, son particularmente exigentes y severas en cuanto a las mujeres». En estas observaciones, Durkheim ya no hace referencia a la limitación natural de los deseos de las mujeres. 
lación social y el suicidio. Al preferir las variaciones del deseo sexual en vez de la obligación social para dar cuenta de la situación antagonista de los sexos en el matrimonio, Durkheim no sólo traiciona su propia ambición de explicar los fenómenos sociales por fenómenos sociales, sino que también deja incompleta su teoría del suicidio.

El contraste entre los dos ejemplos contemporáneos del suicidio fatalista que Durkheim proporciona (las mujeres casadas y los maridos jóvenes) muestra por qué toda explicación basada en la intensidad del deseo está condenada de antemano. Durkheim observa (en el capítulo sobre el suicidio egoísta) que los matrimonios tempranos tienen una influencia aparente en el porcentaje de suicidios masculinos. Al final del capítulo sobre el suicidio anómico explica que se trata de una consecuencia de la regulación excesivamente rígida del matrimonio monógamo: «Sus pasiones son demasiado tumultuosas y seguras de sí como para someterse a una regla tan estricta; esta regla les parece un obstáculo intolerable con el que se enfrentan y se hacen añicos sus deseos» (1897: 309). Durkheim nos había dicho unos renglones antes que si «el hombre, en cierto momento de su vida, [se ve] afectado por el matrimonio del mismo modo que la mujer» es "por razones distintas». En efecto, se trata de razones completamente distintas e incluso, aunque Durkheim tenga cuidado de no señalarlo, de razones opuestas. El matrimonio parece constituir una obligación excesiva para las mujeres porque sus deseos sexuales son limitados y para los hombres jóvenes porque los suyos son demasiado intensos. El que Durkheim amalgame en el mismo concepto de suicidio fatalista esas dos situaciones conflictivas demuestra la escasa importancia que da a este cuarto tipo de suicidio. Esto no es todo: es evidente que, sobre este punto central de la teoría de la regulación, es decir, la relación entre deseos y reglas, no son las reglas las que varían y aparecen como una variable independiente, sino los deseos.

Por tanto, es patente el carácter incompleto de la teoría de la regulación social y extremadamente difícil, sobre la base de elementos dispares y de los ejemplos en apariencia contradictorios proporcionados por Durkheim, reconstruir un concepto de fatalismo que encaje con su marco teórico general. El concepto que aparece al principio de la nota sobre el suicidio fatalista anteriormente citada es la «regulación excesiva». A este respecto, el cuarto tipo de suicidio "se opone al suicidio anómico exactamente como el suicidio egoísta al suicidio altruista». Pero las notas que siguen no concuerdan con esto y, además, describen una situación tan extrema que es difícil encontrar ejemplos: "personas cuyo futuro está despiadadamente bloqueado», "pasiones violentamente reprimidas por la disciplina opresiva" (lo que precede al ejemplo de los «maridos que son demasiado jóvenes»), «excesivo despotismo físico o moral» y "la ineluctable e inflexible naturaleza de la regla» (1897: 311).

Durkheim intentó ilustrar este concepto con tres ejemplos: los maridos demasiado jóvenes, las mujeres casadas sin hijos y "los suicidios de esclavos, frecuentes en ciertas condiciones». Las reglas son sin duda excesivamente rígidas en todos esos casos; pero ¿es realmente decisiva esta rigidez excesiva cuan- 
do sólo se experimenta en relación a la intensidad de la pasión, como se ve en la relación conyugal? ¿No sería otro el principio del fatalismo? Autores más recientes, como Dohrenwend, encontraron la causa en el carácter externo del poder regulador en relación al grupo y los individuos que lo componen (Dohrenwend, 1959). Esta interpretación podría aplicarse al caso de los suicidios de esclavos, o a los otros casos citados por Durkheim, los suicidios de presos (1897: 394) y los suicidios en masa de los judíos en la toma de Jerusalén, que los convertía en «sometidos y tributarios de Roma» (1897: 326). Pero los casos se corresponden mejor con el principio más general de la imposibilidad de interiorizar reglas que son injustas e ilegítimas porque se imponen desde el exterior (esclavos), son vanas (mujeres casadas) o excesivas (maridos jóvenes). Además de que este principio tiene la ventaja de reconciliar los elementos contradictorios del concepto de fatalismo, su aplicación se apoya en un comentario de Durkheim sobre los maridos jóvenes: «Probablemente el matrimonio sólo produce todos sus efectos benéficos cuando la edad ha templado al hombre y le ha hecho sentir la necesidad de la disciplina» (1897: 309-310) ${ }^{3}$.

Si el núcleo del principio del fatalismo es la imposibilidad de interiorizar una regulación inaceptable, su concepción es muy cercana al contraste entre la división anómica del trabajo y la división forzosa del trabajo. «Las reglas por sí no bastan, deben ser además justas», escribirá Durkheim sintetizando la naturaleza opuesta de estas dos formas patológicas de la división del trabajo en las páginas finales de su tesis (1893: 403). La segunda forma «se mantiene sólo por la fuerza» y la "constricción». La constricción impide que los individuos «ocupen un lugar en la jerarquía social acorde a sus capacidades» (1893: 369371)

Si aceptamos esta interpretación del fatalismo, quedamos todavía en manos de ambigüedades que nos impiden llegar a una teoría de la regulación social coherente y clara. La primera dificultad visible está en conexión con el comentario de Durkheim sobre los efectos variables del matrimonio dependientes de la edad. ¿Se beneficiaría el hombre maduro y "temperado» de la disciplina conyugal - o, por el contrario, sufriría si se debilitase - si ha interiorizado realmente la necesidad de la obligación conyugal? ¿Es la disminución del deseo la condición necesaria para la interiorización de la norma? Como la situación es muy diferente para las mujeres, esta interpretación del fatalismo choca con un problema adicional: a no ser que supongamos que hay un término medio del deseo favorable a la aceptación de la regla de la monogamia, estamos obligados ya sea a formular un postulado nuevo, menos económico y menos plausible, ya a abandonar completamente cualquier intento de interpretar el antagonismo entre los sexos en el matrimonio por la intensidad del deseo. Podríamos considerar, por ejemplo, que la constricción es excesiva para las mujeres casadas porque se superpone a otras ligadas a su estatus subordinado.

Centrar la atención en la imposibilidad de interiorizar una norma como el

${ }^{3}$ Durkheim considera «que probablemente el matrimonio en sí mismo no empieza a tener un efecto profiláctico hasta... pasada la edad de treinta años» $(1897: 310$, n. 1) 
rasgo fundamental del fatalismo provoca otra dificultad, y no la menos seria. Esta interpretación subraya lo que tienen en común la anomia y el fatalismo, y no las diferencias que los oponen. La razón de esta ausencia de interiorización de las normas — su debilidad en el caso de la anomia, su ilegitimidad en el del fatalismo - es sin duda diferente; pero no opuesta. Podríamos incluso preguntarnos si el fatalismo no sería más que un tipo particular de anomia: parece íntimamente relacionado con lo que Durkheim llama "anomia regresiva", donde el carácter intolerable de la norma se debe a su inadaptación a las condiciones presentes. Éste sería el caso de los individuos que experimentan «una auto-represión creciente» como resultado de una pérdida de estatus (1897: 280). O, por el contrario, ¿esta forma de anomia estaría subsumida en el fatalismo?

Responder a estas dos preguntas requeriría un examen detallado de los rasgos y condiciones de la anomia, al que no procederemos. Aun así, ya es evidente que el olvido de Durkheim del suicidio fatalista deja la teoría de la regulación social incompleta y confusa y, en consecuencia, impide llegar a una comprensión precisa de la noción de anomia.

\section{ANOMIA AGUDA Y ANOMIA CRÓNICA}

La anomia se presenta constantemente como una dicotomía, subdividida en dos modalidades. Durkheim analiza dos formas de anomia en particular, la económica y la conyugal (esta última debe distinguirse de la anomia doméstica, originada por la crisis de la viudez, y de la anomia sexual, que es la anomia de los no casados). Durkheim utiliza dos contrastes adicionales, entre anomia aguda y anomia progresiva. ¿Hay un principio común subyacente a estas diferentes modalidades o incluso alguna clase de lógica que permita unirlas?

La distinción entre anomia "aguda» y anomia "crónica» domina los análisis de la anomia económica. Durkheim empieza por estudiar el impacto de las crisis económicas y el efecto perjudicial que tienen sobre el porcentaje de suicidios antes de contrastar esta anomia, que surge "a rachas y en crisis agudas», con la anomia "en un estado crónico», que es un "factor constante y regular» del suicidio (1897: 282-283) ${ }^{4}$.

Las crisis económicas, ya sean "catástrofes económicas» o, en el caso contrario, "crisis de prosperidad", en otras palabras "transformaciones beneficiosas pero demasiado bruscas» $(1897: 271,280)$, son el primer y principal ejemplo de anomia aguda dado por Durkheim. En ambos casos se interrumpe temporalmente el orden colectivo. La anomia preponderante en «la esfera del comercio y de la industria» se encuentra al mismo tiempo "en un estado crónico» (1897: 283). Pero la anomia crónica en este sentido es intrínseca al progreso

4 "Aguda" significa aquí "transitoria», en contraste con estructural o permanente (crónica). No se trata de una forma extrema de anomia, como es el caso en la terminología de De Grazia (1948). 
económico característico de las sociedades modernas, cuyo objetivo final es la prosperidad industrial.

«La liberación de los deseos» y «la efervescencia» características del comercio y la industria se extienden al resto de la sociedad. "El estado de crisis y de anomia — sigue Durkheim - es constante y, por decirlo así, normal aquí» (1897: 285, itálica añadida). Expresiones tales como anomia crónica o anomia estructural son sin duda demasiado débiles para describir el caso en que la anomia está virtualmente institucionalizada en el sistema de valores de la sociedad moderna, en cuyo corazón reside. Si es algo "normal» en estas sociedades, es porque son patológicas.

No podemos insistir lo suficiente en que el concepto de anomia implica una condena vigorosa y casi vehemente de la ideología de la sociedad industrial. En un pasaje típico, Durkheim revela la fuerza de convicción que le motiva a través de las florituras estilísticas que adornan las secciones de El Suicidio dedicadas a la anomia.

«Estas disposiciones son tan inveteradas que la sociedad las ha aceptado y se ha acostumbrado a considerarlas normales. Se repite sin cesar que el hombre está por naturaleza eternamente descontento y avanza siempre, sin tregua y sin descanso, hacia un fin indeterminado. La pasión del infinito se presenta diariamente como una marca de distinción moral, cuando sólo puede producirse en el seno de conciencias desordenadas que erigen en regla el desorden que sufren. La doctrina del progreso a cualquier precio y lo más rápido posible se ha convertido en un artículo de fe» (1897: 286-287).

Esta dualidad entre la anomia de crisis y la anomia institucionalizada se relaciona con la familia y el matrimonio, así como con el análisis de la anomia económica. La anomia de crisis resultante de la transición brusca de un estado a otro (como la viudez o el divorcio) contrasta con la anomia conyugal que afecta a los hombres casados, «inscrita en la ley» por la institución del divorcio. Este ejemplo muestra que la anomia puede institucionalizarse, en el sentido estricto del término, desde el momento en que «se convierte en una institución legal» (1897: 307).

Pero este ejemplo nos enseña también que las dos modalidades de la anomia no son tratadas de igual manera por Durkheim: otorga más importancia a la anomia crónica que a la anomia aguda. Tras algunos renglones sobre la crisis de la viudez y la anomia doméstica resultante, explica inmediatamente que va a dedicar más espacio a la forma de anomia (anomia conyugal) "más crónica» (1897: 290). De manera similar, no se interesa por las consecuencias directas del divorcio sobre el individuo; menciona este "cambio en la situación moral e intelectual» sólo para afirmar que no constituye una explicación adecuada (1897: 294). No hay una sola alusión a la anomia en El Suicidio que pueda asociarse a otro cambio de estatus: el matrimonio mismo. Sin duda, era difícil 
demostrar la influencia sobre el suicidio de este estado de transición de la anomia sobre la base de cuadros preparados según los grupos de edad. Pero, utilizando los informes individuales sobre el suicidio, Durkheim hubiera podido comparar las fechas de los suicidios con las fechas de los matrimonios o utilizarlos para explicar los suicidios relativamente frecuentes entre los maridos muy jóvenes. Cuando le conviene, emplea, sin problema, los datos empíricos con exceso. Pero lo hace en contextos donde critica lo que le interesa, como los ejemplos de la anomia conyugal institucionalizada que afecta a los maridos y de la "anomia sexual» que los no casados "viven de manera crónica» (1897: 308).

Al final, se nos dan algunas ilustraciones de anomia aguda o transitoria en El Suicidio: el cambio brusco en la situación económica, la anomia doméstica, y otro cambio repentino, la jubilación de los oficiales y suboficiales (1897: 326). Aunque Durkheim toma el ejemplo de las crisis económicas como su punto de partida empírico, la anomia crónica está al frente de sus preocupaciones, a pesar de la falta de datos relevantes respecto de la anomia económica. Sus motivos son aquí transparentes: desea mostrar que «en nuestras sociedades modernas» la anomia no es sólo un factor "específico», sino también "habitual», «una de las fuentes que suministra el contingente anual» de suicidios (1897: 288).

Sin embargo, iríamos demasiado lejos si afirmáramos que Durkheim considera la anomia aguda como un fenómeno insignificante, o que esas dos formas de anomia son intercambiables. Las dos modalidades son diferentes. Pero no carecen de lazos, ya que, de acuerdo con el mismo Durkheim, la anomia crónica fomenta la aparición de la anomia aguda. Las catástrofes económicas son «tan fértiles en suicidios» a causa del «estado moral» de anomia crónica en el que surgen, y, como «los fracasos aumentan con los riesgos», su número aumenta (1897: 285-286). La anomia está sin duda ligada, en ambas modalidades, al cambio. Pero en un caso se trata del fruto de un cambio brusco en el mundo social y en el otro resulta de una situación en que el cambio y la ausencia de referencias caracterizan el contexto social del individuo.

\section{ANOMIA REGRESIVA Y ANOMIA PROGRESIVA}

Otra dualidad de la anomia explícita en El Suicidio es la dicotomía entre anomia «regresiva» y anomia "progresiva». Esas expresiones sólo aparecen en el capítulo sobre las formas individuales de los tipos de suicidio (1897: 322). Pero la distinción informa el análisis de la anomia económica desde el principio. El capítulo sobre el suicidio anómico comienza con la constatación de un "hecho conocido", "las crisis económicas tienen un efecto agravante sobre la propensión al suicidio» (1897: 267) —o al menos cree poder afirmarlo legítimamente, a pesar de la fragilidad y de la disparidad de los datos empíricos utilizados-. Primero, Durkheim nos invita a deducir el factor anómico del 
aumento de suicidios sobre la base de lo que tienen en común estas dos situaciones simétricas - que son "crisis, esto es, interrupciones del orden colectivo"- (1897: 271). En ambos casos, la sociedad es temporalmente incapaz de cumplir su acción reguladora sobre los deseos individuales (1897: 280); en ambos casos, las necesidades se liberan "de la moderación adecuada» (1897: 322). La simetría entre anomia «regresiva» y "progresiva» es completa, o así se ha interpretado habitualmente, y, en efecto, una lectura superficial de ciertas páginas de El Suicidio puede inducir a tal interpretación. En mi opinión, esta concepción de la anomia no resiste un análisis minucioso. Al contrastar la anomia regresiva y la anomia progresiva, Durkheim se ha limitado a un esbozo engañoso de trompe-l'oeil por amor de la simetría.

Los pasajes concernientes a la anomia progresiva están mucho más elaborados que los dedicados a la anomia regresiva. La palabra anomia aparece por vez primera en El Suicidio (1897: 281) respecto de las crisis que surgen del bienestar creciente, y, no sin sorpresa, Durkheim se sirve de este fenómeno para continuar su crítica del utilitarismo. La simetría establecida entre las crisis de depresión y las de prosperidad debe entenderse en el contexto de una prueba específica. El paralelo introducido por Durkheim entre las crisis económicas, en el sentido normal del término, y las crisis de prosperidad es principalmente un medio de refutar la idea, generalmente aceptada, de que el empobrecimiento es en sí mismo causa de suicidio. Durkheim insiste en que «la angustia económica», lejos de tener un efecto agravante sobre el suicidio, "tiene más bien un efecto positivo" (1897: 269) y, para probarlo empíricamente, compara las categorías de los Departamentos clasificados por porcentaje de suicidios y la proporción de población con recursos independientes en cada categoría de los Departamentos.

El paralelo entre las dos formas va más allá de esta prueba. Pero, por mucho que lo intente, Durkheim tiene muchas dificultades para preservar su simetría. El pasaje dedicado a las formas individuales del suicidio anómico, donde aparecen las expresiones «regresiva» y "progresiva», es confuso. "Progresiva o regresiva, la anomia, al liberar las necesidades de la moderación adecuada, abre la puerta a las ilusiones y en consecuencia a la desilusión» (1897: 322). Pero ¿cómo podría la anomia regresiva ser una fuente de ilusiones? En el ejemplo que da Durkheim de un "hombre que bruscamente retrocede en relación con su estatus habitual» podemos ver cómo habría «desilusión» y «exasperación", pero las ilusiones y la desilusión consecuente sólo proceden de la anomia progresiva y de la liberación de la moderación que implica.

Durkheim pasa rápidamente por encima de la anomia regresiva para concentrarse en la anomia progresiva, en que "por el contrario, sin regla ni moderación, el individuo está continuamente incitado a superarse» (1897: 322). Distingue tres situaciones típicas de anomia progresiva que conducen al suicidio. En el primer caso, el individuo «no obtiene el fin que creía poder alcanzar (...); es el suicidio del incomprendido, tan común en las épocas en que no hay una clasificación social reconocida». Durkheim no proporciona más detalles de 
este tipo de anomia progresiva, dejándonos un poco perplejos. La lógica subyacente a este vínculo entre la ausencia de una clasificación reconocida y el exceso de aspiraciones en relación con las capacidades, y el hecho de la «incomprensión no es evidente». El segundo tipo de situación individual, el individuo que ha "conseguido por un tiempo satisfacer todos sus deseos y su amor por el cambio, [pero que] de repente choca con un obstáculo invencible», es descrito e ilustrado con gran detalle. No sólo las aspiraciones de este individuo son elevadas, sino que también han sido en gran parte alcanzadas; un obstáculo que surge en el curso de esta curva ascendente puede conducir al individuo al suicidio. El obstáculo no tiene por qué ser muy importante. Además del ejemplo de Werther (discutible), «enamorado del infinito y que se da la muerte porque se ve frustrado en amor", Durkheim ilustra el riesgo psicológico de la curva ascendente del éxito con ejemplos de «artistas que, después de haberse mostrado con éxito, se suicidan porque corren el riesgo de escuchar un comentario desdeñoso, por causa de una crítica lo bastante dura, o porque su popularidad cesa de aumentan» (1897: 322-323, itálica añadida).

La tercera "forma individual» tratada por Durkheim es, incontestablemente, la forma más típica de la anomia progresiva. Concierne a los individuos que «aunque no tienen quejas de los demás o de las circunstancias, se aburren de una carrera sin conclusión posible y donde sus deseos se exacerban en vez de apaciguarse» (1897: 323). En este capítulo los ejemplos preferidos de Durkheim son los personajes literarios, aunque también alude a las observaciones de casos de Bierre de Boismont. Encuentra que el René de Chateaubriand es la personificación más perfecta de este tipo de mentalidad. En contraste con el Rafael de Lamartine («el tipo ideal del suicidio egoísta»), una "criatura meditativa» que "se pierde en sí misma», René es "insaciable», tal como muestran sus quejas, citadas por Durkheim.

«Se me acusa de dejar pasar siempre la meta que puedo alcanzar: ¡ay!, solamente busco un bien desconocido cuyo instinto me persigue. ¿Es culpa mía si encuentro en todas partes límites, si lo que está acabado no tiene para mí ningún valor?»(1897: 323-324).

Más tarde, el Fausto de Goethe ofreció a Durkheim «la personificación absoluta de este sentimiento del infinito». Durkheim vio en éste una característica del tiempo presente, que es triste porque «el pesimismo siempre va de la mano de las aspiraciones ilimitadas» (1925: 35) . Éste es el núcleo del tratamiento de

En El Suicidio $(304$, n. 2) se menciona Fausto para ilustrar la enfermedad del infinito. En el mismo pasaje, Durkheim hace una alusión fugaz a dos ejemplos de anomia sexual que toma prestados de Musset: Rolla y «el retrato de Don Juan» en Namouna. Si Durkheim pasa por encima de estos dos ejemplos tan rápido, quizás sea porque no encajan exactamente en su argumentación. Rolla (quien, en verdad, se suicida) y Hassan, el protagonista de Namouna, son figuras románticas que viven una especie de doble vida: por un lado, una vida de placer inmediato y desenfreno y, por otro, la búsqueda de un amor único y total, el anhelo de la pureza perdida. A mi parecer, estos personajes ilustran la búsqueda romántica de un absoluto imposible más que 
la anomia de Durkheim. Los hombres están destinados a perderse en la infinidad del deseo, ya que la incertidumbre de los objetivos que hay que alcanzar significa que el «punto final (...) se escapa progresivamente a nuestro alcance a medida que avanzamos» (1925: 35). El carácter ilimitado de las aspiraciones conduce necesariamente a la frustración y al tormento.

En estas tres situaciones típicas de la anomia progresiva descritas por Durkheim, la insatisfacción nace en un contexto de movilidad ascendente apropiado para alumbrar ilusiones que posteriormente se harán añicos. Pero ¿es diferente la anomia regresiva? Los repentinos hundimientos económicos, o, en el caso de los individuos, un inesperado revés de la fortuna, son accidentes que surgen sobre un fondo de anomia crónica progresiva. A este respecto, no hay una radical discontinuidad entre la regresión repentina y el fenómeno por etapas anteriormente citado. Por lo tanto, al tratar de sus manifestaciones individuales, Durkheim no logra distinguir entre la anomia regresiva y la anomia progresiva.

Esta dicotomía es totalmente inoperante en los casos de la anomia conyugal o anomia sexual, que radica completamente en la esfera de la anomia progresiva. Pero ¿es apropiado tratar la anomia conyugal de progresiva? ¿No es la indeterminación de los fines, en lugar del progreso hacia un objetivo que constantemente escapa a nuestro alcance, la característica central aquí? Para sentir la exasperación característica de la anomia, Durkheim observa que no es necesario "haber tenido un número infinito de experiencias amorosas y vivido la vida de Don Juan. La existencia rutinaria del soltero ordinario basta», puesto que le condena a la «movilidad perpetua» (1897: 305). Pero es fácil ver que esta objeción potencial no es válida. La indeterminación de las metas y la ilimitación de las aspiraciones están íntimamente ligadas a ojos de Durkheim. El matrimonio "cierra el horizonte» y «limita el deseo» porque «asigna un objeto definido con precisión a la necesidad de amar». Pero cuando el vínculo conyugal puede romperse, "no se puede sino mirar más allá de la situación presente» (1897: 304-305).

La estrecha proximidad de ambas nociones, su superposición en realidad, es además un leitmotif que atraviesa numerosas páginas de L'éducation morale. Para evitar la "enfermedad del infinito", la actividad humana debe tener siempre un objetivo específico «que la limite determinándola» (1925: 35). El propósito de la disciplina es «asignar fines específicos que, al mismo tiempo, limiten su horizonte» (1925: 41). "Sólo a través de la disciplina puede enseñarse a un niño a limitar sus apetitos y, al hacerlo, definir los objetivos de su actividad» (1925: 38, itálica añadida). Lo que sugiere claramente que la ilimitación del deseo no puede distinguirse de la indeterminación del objeto de dicho deseo. Así, la anomia sexual o conyugal debe considerarse como una forma de anomia

un deseo de infinito, como lo describe Durkheim. Su característica fundamental no es tanto la indeterminación del objeto deseado que el apego a un objeto ideal inaccesible pues irreal o mítico. 
progresiva, una razón adicional para tratar la anomia progresiva como la verdadera anomia.

\section{TIPOLOGÍA DE REGULACIÓN}

De esta manera no es posible definir la noción de anomia como el núcleo común de ambos tipos, progresiva y regresiva. La simetría entre las dos modalidades sugerida por Durkheim en ciertas páginas de El Suicidio es, de hecho, ilusoria. Cuando Durkheim describe las situaciones individuales típicas que conducen al suicidio, está claro lo que ambos casos tienen en común: no es más que los antecedentes de la anomia progresiva. La experiencia del fracaso individual es tan dolorosa porque las aspiraciones eran muy elevadas, por no decir ilimitadas. Del mismo modo, las catástrofes económicas son letales sólo en virtud del anhelo de infinito característico de las sociedades modernas. Como cualquier límite se considera "detestable», un «límite más estricto» parece intolerable: "cuando no se tiene otra intención que superar constantemente el punto alcanzado (...) la obligación de retroceder de nuevo es dolorosa» (1897: 286).

La ausencia de simetría entre las dos «modalidades» de la anomia se revela de otra manera. La anomia regresiva en las discusiones de Durkheim es siempre aguda. No puede haber algo como una anomia regresiva crónica. Por consiguiente, una interpretación literal de El Suicidio no nos permitiría reconstruir una tipología subyacente basada en un cuadro cuádruple completo utilizando las dicotomías "crónica-aguda» y "progresiva-regresiva».

Sin embargo, existe una manera de dar un mayor grado de coherencia a la teoría de la regulación de Durkheim. Para hacerlo, debemos dejar de considerar la anomia regresiva como una forma de anomia: más bien es, en relación al fatalismo, lo que la anomia progresiva en relación a la anomia progresiva crónica. Lo que Durkheim escribe a propósito de los individuos víctimas de una pérdida de estatus de resultas de un desastre económico o de un revés justifica semejante interpretación. "No se ajustan a la condición impuesta» y esta "autorepresión creciente» es una perspectiva «intolerable» para ellos. Afrontan una situación que consideran injusta e ilegítima: «su educación moral debe comenzarse de nuevo" (1897: 280). ¿No son éstos los rasgos principales del fatalismo? Existe, ciertamente, la imposibilidad de interiorizar nuevas normas demasiado coactivas en relación a sus aspiraciones (como en el caso de los maridos demasiado jóvenes) consideradas inaceptables. La pérdida del estatus puede incluso compararse a la esclavitud, al menos si consideramos la situación de los que acaban de ser reducidos al estado de esclavitud. En ambos casos, las aspiraciones profundamente interiorizadas resultantes de la socialización prolongada entran en conflicto con las nuevas normas que sólo pueden considerarse ilegítimas. Otra característica del fatalismo se encuentra en lo que Durkheim califica de anomia regresiva: el sentimiento de no controlar el propio destino [«la regla 
frente a la que se es impotente» (1897: 311)]. El hombre bruscamente desclasado experimenta cómo "una situación que siempre creyó dominar escapa a su control» (1897: 322). En la anomia regresiva, como en el fatalismo, el horizonte de posibilidades está cerrado, el "futuro está despiadadamente bloqueado» (1897: 311) y los deseos "chocan y se hacen añicos» contra "un obstáculo intolerable» (1897: 309)

Estamos ahora en posición de responder a la pregunta planteada anteriormente. Hemos probado la imposibilidad de reducir el fatalismo a una modalidad de la anomia. En su lugar, nuestra conclusión es que la anomia regresiva pertenece al fatalismo. Cuanto más pura y precisa es la noción de anomia, mayor es la esfera de aplicación de la noción de fatalismo. Durkheim trató de reducir al mínimo el papel del fatalismo. Pero, aun sin que se diera cuenta, su presencia es mucho más evidente en sus escritos de lo que sugieren las primeras apariencias y, lejos de confinarse a una mera nota a pie de página, sustenta una gran cantidad de pasajes del capítulo sobre el suicidio anómico 6 .

Todos los problemas de la teoría de la regulación social de Durkheim no se resuelven por este reconocimiento, ni se suprimen todas sus ambigüedades. Pero esta reconstrucción proporciona al menos un grado de coherencia a la teoría sin contradecirla. Entre otras cosas, permite elaborar una tipología que combina dos aspectos: la regulación como tal, que lleva consigo la apertura o el cierre del horizonte de posibilidades (anomia/fatalismo), y la dicotomía entre situaciones crónicas o institucionalizadas y crisis agudas producidas por un cambio repentino. Ver gráfico 7.2.

\section{GRÁFICO 7.2}

Regulación respecto de las situaciones crónica y aguda

\begin{tabular}{lcc} 
& \multicolumn{2}{c}{ Regulación } \\
\cline { 2 - 3 } & $\begin{array}{c}+ \\
\text { Anomia }\end{array}$ & $\begin{array}{c}+ \\
\text { Fatalismo }\end{array}$ \\
\cline { 2 - 3 } & 1 & 2 \\
Forma crónica & 3 & 4
\end{tabular}

${ }^{6}$ Además de las páginas que tratan de las mujeres casadas sin hijos y de los maridos demasiado jóvenes (1897: 306-307, 309-311), y además de los pasajes relativos a la anomia regresiva (280, 285-286), hay un pasaje en que Durkheim observa que, para ser efectiva, una regulación debe ser "considerada justa» y debe, por tanto, venir de un poder al que «se obedece por respeto, no por miedo" (1897: 279). Quizás habría que incluir también en el fatalismo la forma mixta del suicidio "anómico-altruista», bajo la que Durkheim describe casos de anomia regresiva: el "hombre arruinado", «los suicidios en masa», en particular el de los judíos durante la captura de Jerusalén; «la victoria de los romanos, al hacerles súbditos y tributarios de Roma, amenazaba con transformar la clase de vida a la que estaban acostumbrados» (1897: 326). 
El fatalismo, como la anomia, aparece aquí en la forma crónica (casilla 2) y en la forma transicional aguda (casilla 4). Esta última resulta del cierre brusco del horizonte de posibilidades y la imposición repentina de normas excesivamente coactivas o inaceptables. Hemos visto que el «fatalismo agudo» no es difícil de ilustrar, aunque nos limitemos a los ejemplos dados en El Suicidio: las víctimas de la crisis económica, las personas reducidas a la esclavitud o los pueblos sometidos, y los hombres recién casados. Las crisis de prosperidad ilustran la casilla 3. La casilla 1 parece corresponder a la anomia que, en El Suicidio, Durkheim sitúa en el centro de sus intereses: la pérdida de sí en la infinidad del deseo, la sensación de vértigo al confrontarse con la apertura ilimitada del horizonte de posibilidades. Esta anomia, inseparable de «toda la ética del progreso y del perfeccionamiento" (1897: 417), se encuentra en el corazón del sistema de valores de las sociedades modernas y es, en efecto, parte esencial de sus instituciones.

\section{LA UNIDAD DE LA ANOMIA}

La interpretación de la teoría de la regulación social en El Suicidio sugerida aquí se caracteriza por depurar la noción de anomia de las connotaciones secundarias al reducir el número de sus posibles modalidades. Sin embargo, la distinción entre anomia aguda y crónica permanece, y puede incluso expresarse por una antítesis. En efecto, la anomia aguda se refiere a la ausencia temporal de normas, o a una falta de normas adaptadas a la situación nueva: «toda regulación está ausente por un tiempo. Ya no se sabe lo que es posible y lo que no, lo que es justo y lo que es injusto", como escribe Durkheim a propósito de esas crisis cuyo origen es "un brusco incremento del poder y del bienestar " (1897: 280). La anomia crónica, por el contrario, se refiere a la presencia, en la cultura moderna, de la ideología del progreso a toda costa. Nos encontramos así frente a dos factores determinantes de la anomia que no deben confundirse. Aunque suprimamos la anomia regresiva de la categoría de la anomia aguda, los dos tipos producen los mismos resultados. En la anomia aguda, como en la crónica, «no hay límite a las aspiraciones» y «los apetitos (...) ya no conocen los límites en que deben pararse» (1897: 281). La anomia procede así de dos condiciones sociales que el análisis debe distinguir, aunque sus efectos respectivos estén interrelacionados y sean recíprocos; no obstante, el término indica el mismo fenómeno: el carácter ilimitado del deseo y la indeterminación de su objeto.

Esta interpretación se separa, en términos nada inciertos, de numerosos analistas de Durkheim que han pensado poder discernir, en El Suicidio, dos conceptos de anomia muy diferentes. De acuerdo con La Capra (1972: 159162), la anomia puede indicar un estado de completa ausencia de regulación y sentido tras un hundimiento del sistema normativo que se expresa en un sentimiento de frustración y angustia, pero también puede indicar la existencia de 
desequilibrios extremos en el sistema social (falta de armonía entre los medios y las necesidades o las aspiraciones). Se asemeja así a la noción marxista de "contradicciones estructurales». Por lo tanto, "desde el punto de vista de Durkheim, la explotación podría considerarse como una variante de la anomia» (La Capra, 1972: 161). Para justificar esta afirmación, La Capra se refiere al pasaje citado más arriba donde Durkheim declara que la disciplina sólo es útil si se la considera justa y si no es mantenida únicamente por la fuerza. Si una situación de este tipo, que según la interpretación que damos se sitúa en el ámbito del fatalismo, se describe en términos de anomia, debemos, en efecto, atribuir una connotación amplia y vaga al concepto de anomia de Durkheim. Por eso no es sorprendente que La Capra emplee el término alienación (1972: 162) como equivalente de anomia.

La interpretación que propone Giddens (1971: 224; 1978: 107) se acerca a la de La Capra, aunque su Durkheim esté más próximo de Merton que de Marx. En su opinión, Durkheim utiliza el término anomia para indicar dos fenómenos diferentes: 1) la ausencia de normas coherentes que definan un objetivo claro para los individuos, y 2) el hecho de alcanzar un objetivo dado. Giddens afirma, sin pruebas, que, a pesar de que esta segunda connotación aparezca con menos frecuencia que la primera en las discusiones teóricas sobre la anomia, está delineada en los análisis empíricos de Durkheim. Semejante interpretación es injustificada, no sólo porque desvirtúa la noción de fatalismo, sino porque también, al contrario de lo alegado por Giddens, es totalmente inadecuada para comprender los análisis empíricos de Durkheim. En el caso de la anomia sexual, por ejemplo, está claro que no es el carácter inaccesible del objeto de deseo lo que caracteriza la anomia, sino su indeterminación.

Estas dos lecturas de El Suicidio nos sitúan frente al mismo hecho. El estado incompleto de la teoría de la regulación de Durkheim, evidente en su subestimación del alcance del fatalismo, contiene las semillas de las metamorfosis que la anomia sufrirá posteriormente en la sociología. Por otro lado, la interpretación que hemos presentado, aunque pueda deformar ciertos pasajes de El Suicidio (por la eliminación de la anomia regresiva, por ejemplo), da un significado más restringido y preciso del concepto de anomia, libre de contradicciones internas y de acuerdo con los datos empíricos del libro y la intuición fundamental de Durkheim.

\section{BIBLIOGRAFÍA}

Besnard, P. (1973): "Durkheim et les femmes ou Le suicide inachevé», Revue Française de Sociologie, 14: 27-61.

- (1987): L'anomie: ses usages et ses fonctions dans la discipline sociologique depuis Durkheim, París: Presses Universitaires de France.

De Grazia, S. (1948): The Political Community: A Study of Anomie, Chicago: University of Chicago Press.

DOHRENWEND, B. P. (1959): «Egoism, altruism, anomie and fatalism: a conceptual analysis of Durkheim's types», American Sociological Review, 24, 4: 466-472. 
Durkheim, E. (1888): «Suicide et natalité: étude de statistique morale», Revue Philosophique, 26 : 446-463.

- (1893): De la division du travail social, Paris: Alcan (las páginas citadas en el texto corresponden a la segunda edición, de 1902, idéntica a las ediciones posteriores).

- (1897): Le suicide: étude de sociologie, París: Alcan.

- (1906): «Le divorce par consentement mutuel», Revue Bleue, 44, 5: 549-554.

- (1909): Texto de contribución al debate sobre "Matrimonio y divorcio», Libres entretiens (Union pour la vérité), 5e série, 258-293.

- (1925) L'éducation morale, París: Alcan (las páginas citadas en el texto corresponden a la nueva edición de 1963 de Presses Universitaires de France).

Giddens, A. (1971): "The "individual" in the writings of Emile Durkheim», Archives Européennes de Sociologie, 12: 210-228.

- (1978): Durkheim, Glasgow: Fontana/Collins.

GUYAU, J. M. (1885): Esquisse d'une morale sans obligation ni sanction, París: Alcan.

LA CAPRA, D. (1972): Emile Durkheim. Sociologist and Philosopher, Ithaca, NY: Cornell University Press.

\title{
(Traducción: Amparo LASÉN.)
}

\begin{abstract}
Suicide's theory of regulation is incomplete owing to the underdevelopment of the fatalist suicide type. It is here proposed a new reading of Suicide so as to reconstruct fatalism and purify anomie as general concepts. The outcome is the arising of a systematic and rigorous theory that allows us to distinguish four types of pathological regulation: acute fatalism, chronic fatalism, acute anomie and chronic anomie. The proposed reconceptualization is set up against other interpretations wich do not solve Suicide's reading problems.
\end{abstract}

